\title{
Alt gegen neu
}

NOËMI HENGARTNER In der Neurorehabilitation wird permanent diskutiert, ob die „neuen“ Konzepte den „alten“ überlegen sind. Noëmi Hengartner prüfte nun, welche Therapieform das Gehen effektiver verbessern kann: das „neue“ aufgabenorientierte Training (AOT) oder das „alte“ Bobath.

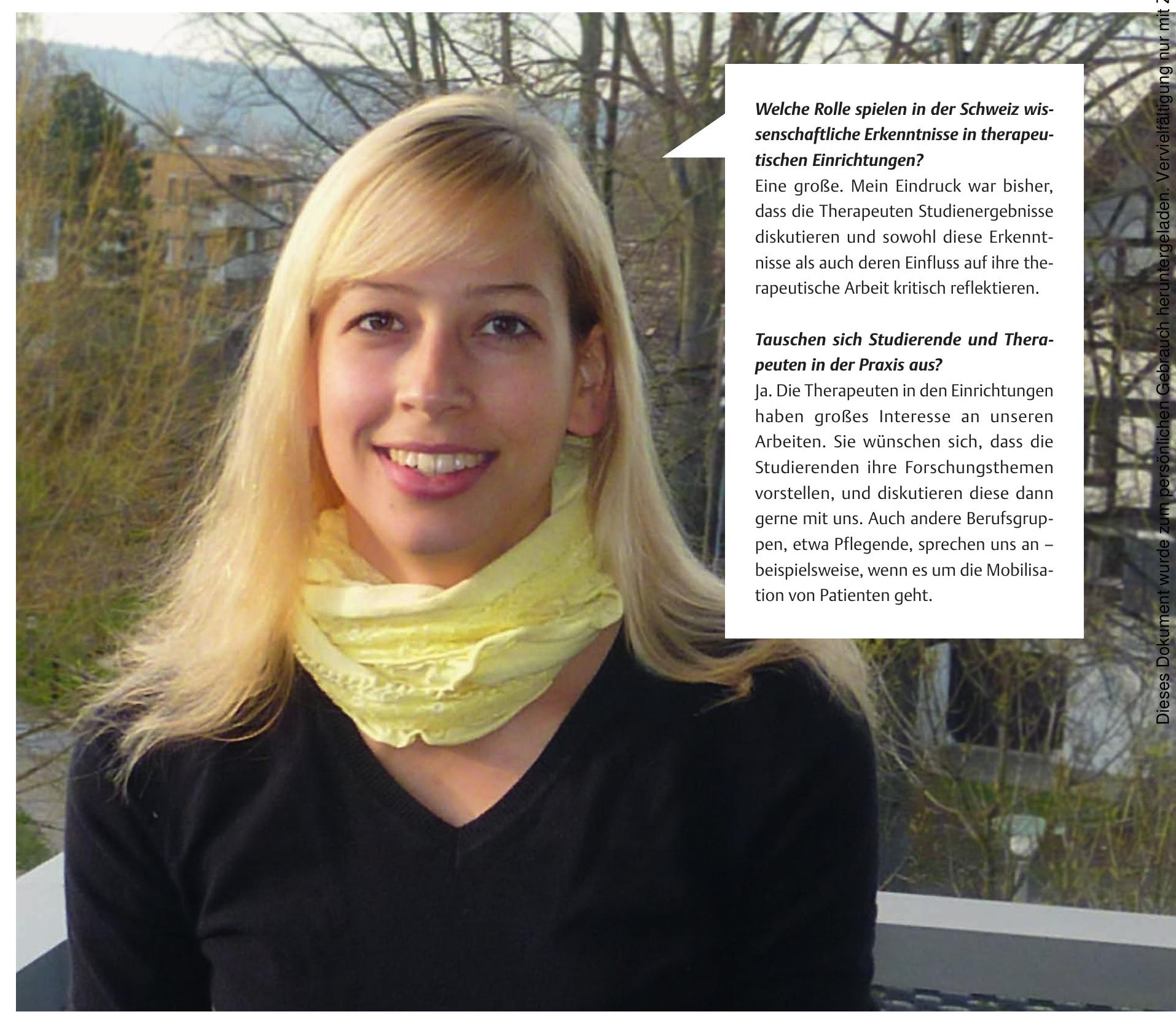




\section{Die Bachelorarbeit: Gangrehabilitation nach Schlaganfall}

Noëmi Hengartner ...

... ist 25 Jahre alt und lebt in Zürich. Seit 2010 besucht sie den vierjährigen, primärqualifizierenden Bachelorstudiengang Physiotherapie der Zürcher Hochschule für Angewandte Wissenschaften in Winterthur. Zwei Jahre davon verbringen die Studierenden in Praktika in verschiedenen therapeutischen Einrichtungen. Nach drei Jahren schreiben sie ihre Bachelorarbeit, im Anschluss arbeiten sie ein Jahr praktisch. Noëmi Hengartner hat sich hierfür ein stationäres Pflegezentrum ausgesucht, wo sie überwiegend Patienten mit neurologischen Erkrankungen behandelt. Die Neurologie und insbesondere das motorische Lernen haben es ihr besonders angetan. Patienten dabei zu begleiten, Bewegungen wieder neu zu erlernen, empfindet sie als schöne Herausforderung. Im Sommer 2014 wird sie ihr Studium beenden. Danach möchte sie Berufserfahrung sammeln, am liebsten in der Neurologie eines kleineren Spitals. In ihrer Freizeit bereist sie sooft es geht andere Länder. Sie liebt es, neue Kulturen kennenzulernen und zu entdecken, wie das Leben in anderen Teilen der Welt funktioniert. Bisher war sie in Asien und Ozeanien unterwegs, zuletzt in Neuseeland. Daheim geht sie gerne wandern und tanzen, am liebsten Ballett.
Nach einem Schlaganfall sind die ersten vier Monate besonders relevant. Denn in dieser Subakutphase ist die neurale Erholung am größten. Es ist also wichtig, dass die Betroffenen in diesem Zeitraum möglichst intensiv rehabilitiert werden.

Eine Grundvoraussetzung für die Teilhabe am sozialen Leben ist die Gehfähigkeit. Diese zu verbessern bzw. wiederherzustellen ist eines der Hauptziele in der Reha nach Schlaganfall. Vor allem zwei Therapieansätze kommen dafür zum Einsatz: das aufgabenorientierte Training (AOT), zum Beispiel Laufbandtraining oder Gehen in der Ebene, und das BobathKonzept. Die Wirksamkeit beider Ansätze wird in Wissenschaft und Praxis kontrovers diskutiert. Aus diesem Grund beschloss Noëmi Hengartner, deren Evidenzlage im Bereich der Gangrehabilitation in ihrer Bachelorarbeit genauer zu untersuchen. Sie wollte vor allem herausfinden, welchen Einfluss beide Ansätze auf die Gehgeschwindigkeit der Betroffenen im Alltag haben. Denn dies gilt als ein Maß dafür, wie stark die Aufgabe in der Therapie dem Umweltkontext ähnelt.

Noëmi Hengartner recherchierte in den Datenbanken MEDLINE und CINAHL nach randomisierten kontrollierten Studien. Von 27 relevanten Arbeiten entsprachen letztendlich vier ihren Einschlusskriterien. Sie erreichten zwischen fünf und acht Punkte auf der zehnstufigen PEDro-Skala und wiesen damit eine mittlere bis sehr gute Qualität auf. Allerdings waren in diesem Fall nur acht von zehn Punkten erreichbar, da eine Verblindung von Therapeut und Patient nicht möglich war. An den Studien nahmen jeweils zwischen 25 und 60 Probanden teil, die Interventionsdauer betrug zwischen zwei und sechs Wochen. In zwei Studien erhielten die Patienten drei Therapieeinheiten pro Woche, in den anderen beiden fünf.

\section{Ergebnisse}

Noëmi Hengartner hat herausgefunden, dass ...

> sich die Gehfähigkeit signifikant und klinisch relevant (um $0,1 \mathrm{~m} / \mathrm{s}$ ) verbessert, wenn die Betroffenen das Gehen funktionell und alltagsnah trainieren.

$>$ keines der beiden Konzepte eindeutig bessere Ergebnisse erzielt.

$>$ tendenziell jedoch das AOT wirksamer ist als das Bobath-Konzept. Sie vermutet, dass der Lerntransfer beim AOT dadurch begünstigt wird, dass in der Therapie mehr Zeit für das effektive Gehen aufgewendet wird, beim Bobath-Konzept hingegen vorbereitende Maßnahmen eine größere Rolle spielen.

$>$ beim AOT laut einer Studie auch Langzeiteffekte nachweisbar waren (Follow-up nach drei Monaten).

\section{Fazit}

Zusammenfassend kann Noëmi Hengartner festhalten, dass ...

> Therapeuten in der Rehabilitation nach Schlaganfall möglichst viel Zeit darauf verwenden sollten, das Gehen intensiv mit den Patienten zu üben.

$>$ ein wichtiges Ziel dabei ist, den kritischen Wert von $0,66 \mathrm{~m} / \mathrm{s}$ bzw. $2,38 \mathrm{~km} / \mathrm{h}$ für auBerhäusliche Gehgeschwindigkeit zu erreichen. Zum Vergleich: Bei gesunden Menschen beträgt die Gehgeschwindigkeit 1,2 bis $1,4 \mathrm{~m} / \mathrm{s}$ bzw. $4,32 \mathrm{~km} / \mathrm{h}$.

$>$ weitere Studien unbedingt Langzeiteffekte und die Auswirkungen auf die Gehfähigkeit im Alltag untersuchen sollten.

Eva Trompetter

$\rightarrow$ Hengartner N. Lerntransfer in der Neurorehabilitation. Bachelorarbeit an der Zürcher Hochschule für Angewandte Wissenschaften; 2013 